\section{Further Support for the AVMA-Approved Rodent Euthanasia Protocol}

\section{David V Gauvin*, Zachary J Zimmermann and Theodore J Baird}

Neurobehavioral Sciences, MPI Research Inc, Michigan, USA

\begin{abstract}
The American Veterinary Medical Association (AVMA) has stated that carbon dioxide is "acceptable with conditions" for euthanasia in those species where aversion or distress can be minimized (Part II, M1.6, p. 24)". With respect to laboratory animals, the AVMA then states, "methods acceptable with conditions" are equivalent to "acceptable methods" when all criteria for application of a method can be met" (Part III, S2, p. 48). This review attempts to provide a unified understanding of the critically-relevant literature bearing on the safety and efficacy of the acceptable conditions required to conduct $\mathrm{CO}_{2}$ euthanasia under the AVMA guidelines. We conclude that $\mathrm{CO}_{2}$ exposure under the AVMA conditions is a rapid, efficient, humane and ethical process of euthanasia in laboratory-bred rodents.
\end{abstract}

Keywords: Alarm calls; Alarm substance; Euthanasia; Rat; Visual function

\section{Introduction}

Due to limited laboratory space, particularly in relation to personnel, equipment needs, and logistical requirements of key study functions it is not uncommon to observe rodent euthanasia procedures being carried out in university, industry, or private research facilities in relatively close proximity to other living laboratory rodents. To the novitiate visiting or working in the laboratory this may pose some emotional dissonance, misgivings or concerns. In consideration of these kinds of emotional reactions, it seems reasonable to take the opportunity to examine some of the empirical evidence establishing a unified and supportable position on the issue of $\mathrm{CO}_{2}$ exposure as an ethical means for laboratory euthanasia of rodents that were not covered in detail within the AVMA euthanasia guidelines. While no one enjoys the process of euthanasia of research animals it is our hope that by understanding the biological, physiological, and psychological

*Corresponding author: David V Gauvin, Neurobehavioral Sciences, MPI Research, Michigan, USA, Tel: +1 2696683336; E-mail: david.gauvin@mpiresearch.com

Citation: Gauvin D, Zimmermann Z, Baird T (2017) Further Support for the AVMA-Approved Rodent Euthanasia Protocol. J Anim Res Vet Sci 1: 002

Received: August 18, 2017; Accepted: September 06, 2017; Published: September 21, 2017

Copyright: (c) 2017 Gauvin D, et al. This is an open-access article distributed under the terms of the Creative Commons Attribution License, which permits unrestricted use, distribution, and reproduction in any medium, provided the original author and source are credited. nature of rodents, the series of events that occur at the end of most every study plan using rodents will viewed as humane and ethical.

\section{Vision}

The actual euthanasia procedure is usually conducted in proximity of the vivarium. The notion or claim that rodents in their home cage are physically capable of bearing witness to euthanasia procedures conducted 10 or 12 feet away within the same testing environment is not consistent with established information regarding the known visual acuity and function of the most commonly utilized laboratory rodents. Rats, for example, are typical mammalian dichromats with short-wavelength cones comprising approximately $10 \%$ of the total retinal cell population [1]. Furthermore, the short-wavelength cones in the rat are most sensitive to ultraviolet range, with a peak at 359 $\mathrm{nm}$. The mid-wavelength cone has peak sensitivity at $510 \mathrm{~nm}$, meaning that rat see best under low ambient light conditions (e.g., at night).

Keeping in mind the background, general information relating to rodent ocular anatomy, it is noteworthy that the preponderance of preclinical toxicity studies are conducted with albino rats. Albino rats are well known to possess relatively poor vision in comparison to pigmented rat strains [2-5]. In a recent study conducted by Kumar et al., [6] Wistar (WI), Sprague-Dawley (SD), Lister-Hooded (LH), and Long Evans (LE) rats were tested for visual acuity using an "Optomotry system" (OptoMotry ${ }^{\mathrm{TM}}$, Cerebral Mechanics, Inc., Lethbridge, Alberta, Canada). While LH and LE rats did not differ in visual acuity, SD and WI rats could not be measured. Although the albino rats did orient towards the screen they failed to display characteristic tracking behaviors in response to the moving gradient under even the "easiest" testing conditions. While human vision of 20:20 corresponds to an acuity of 30 cycles / degree, Kumar et al., [6] concluded that albino WI and SD rats have a visual acuity close to zero cycles per degree. Prusky \& Douglas [7] have concluded that rats have a peak sensitivity of about " 25 " cycles / degree, meaning they can detect $4 \%$ difference in a pattern of 0.1 to 0.2 cycles per degree. Albinism has been reported to be associated with structural abnormalities in the rat visual system including neuro-retinal abnormalities resulting from non-pigmented retinal pigment epithelium, abnormal decussation of retinal ganglion cell axons at the optic chiasm, and abnormal inter-hemispheric connections of the visual cortex [8-10]. According to Prusky \& Douglas [7] the most likely explanation for reduced visual acuity in albino rats is that excessive light scattering within the retina [11], make the eye a rather poor image-forming device and that light-induced retinal degeneration [12] results in poor spatial sampling, as well.

With respect to the influence of conducting $\mathrm{CO}_{2}$ inhalation euthanasia within close proximity to home-cage bound rodents, the practical translation of this information is that in just a two meter distance, the rat can only see vague dark/light shadows. There is sufficient data to conclude that a rat cannot identify shadows or movement 5 to 6 feet from the cage rack. Placing a rat in a chamber to be euthanized 10 to 12 feet from the cage rack, is inconsistent with the available data characterizing the anatomical and operating characteristics of the rodent visual system. Removing rats from their vivarium to conduct $\mathrm{CO}_{2}$ euthanasia will also remove the sounds and smells of study cohorts that may induce states of anxiety and distress. With knowledge of the anatomy and visual sensitivity of rodents, keeping them in the room and allowing euthanasia chambers to be in close proximity to 
the cage racks are consistent with the spirit and intent of the AVMA guidelines.

\section{Alarm calls/vocalizations}

There are two forms of vocalizations in rodents; one audible to humans and one outside the sensitivity range of humans (i.e., ulstrasonic or inaudible).

Like other mammals, rats produce sound through the larynx. But unlike most other mammalian species, rodents can use their larynx in at least two modes of functioning. The first, common to most other species, causes vibrations of vocal folds and produces sounds in the audible range of human technicians (squeeks or squeals) of two to four $\mathrm{kHz}$ [13]. These calls may be expressed by both physical pain and discomfort-acute, approaching, or anticipated [14]. Rats generally direct these vocalizations at humans in anticipation of, or when experiencing, physically unpleasant treatment or rough handling. This "first type" of vocalizations are within the audible range of human hearing.

There is a second mode of larynx function in the rat that is in the emissions of ultrasounds. For these sounds, the larynx is stabilized and used as a whistle with a very small orifice created by the vocal cords [15] which are tightly constricted and cannot vibrate [16]. These ultrasonic vocalizations are NOT in auditory range of human hearing-they remain inaudible to humans. They must be recorded, amplified, and transduced to visual oscilloscopic evidence to observe, enumerate, or quantitatively discriminate. Rats use the ultrasonic call in a variety of social situations that include aversive and appetitive encounters, as summarized below (Figure 1):

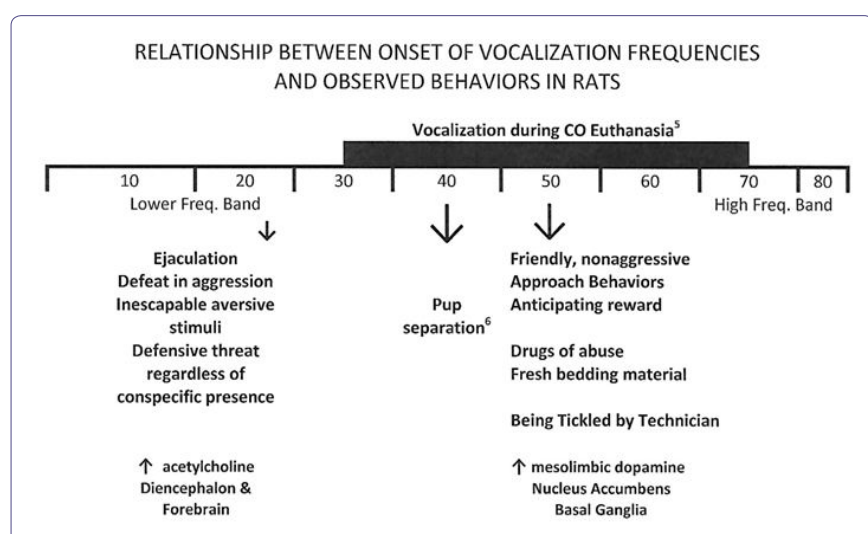

Figure 1: Schematic diagram reflecting the relationship between ultrasonic vocalizations evoked by rats and observed behaviors or behavioral functions observed during those vocalizations. Low ultrasonic frequency bands of $22 \mathrm{kHz}$ reflect aversive/escape situations and mid-frequency ultrasonic bands of $50 \mathrm{kHz}$ reflect appetitive/approach situations.

In 2013, Chisholm et al., [17] reported the presence of ultrasonic vocalizations during exposure to $\mathrm{CO}_{2}$ gas during standard rodent euthanasia approved by the USA Veterinary Association and the Canadian Council on Animal Care guidelines. All nine female Sprague-Dawley rats emitted vocalizations in the ultrasonic range of approximately $50 \mathrm{kHz}$ during exposure to carbon dioxide, but not isoflurane. On superficial examinations of these findings, it might be compelling to propose that such auditory vocalizations are based on perceived threat by the animals in what, anthropomorphically, would seem to be an "anxiety" inducing situation for the rat (Figure 2).

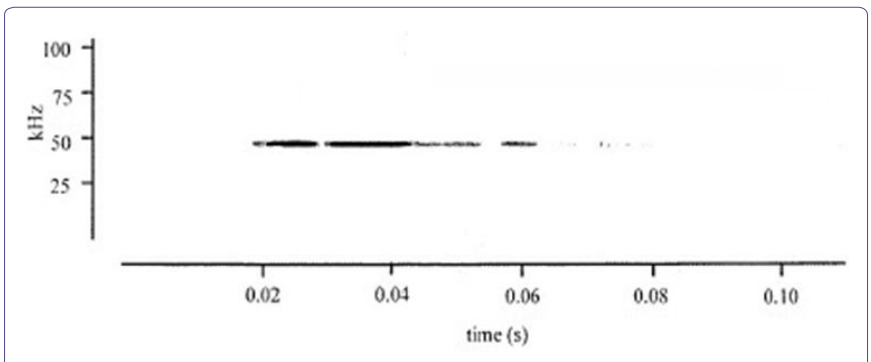

Figure 2: Example of a typical ultrasonic vocalization emitted by a female rat exposed to $100 \%$ carbon dioxide at a fill rate of $30 \%$ volume per minute. All rats exposed to carbon dioxide gas ( 8 out of 8 animals) produced ultrasonic vocalizations. From Chisholm, De Rantere, Fernandez, Krajacic, \& Pang [17]. Source: Reproduced with permission from SAGE publishing.

While it may be true that rodent ultrasonic vocalizations may serve as a sensitive communicative process in a number of rodent-relevant individual and social behaviors, it should not be assumed that the recorded vocalizations reported by Chisholm et al., [17] reflect an "alarm call" to cohorts during euthanasia in the close proximity (same room) as other housed rats.

As shown above, low frequency ultrasonic vocalizations $(22 \mathrm{kHz})$ have been considered to reflect avoidance and aggression and, for some authors, serve as an index of a subjective state comparable to human anxiety, since they are emitted during various unconditioned and conditioned aversive situations [18]. Alcohol withdrawal and the subjective effects of kappa opiates generally produce negative affective states in humans. Both of these drug-associated states in rats induce $22 \mathrm{kHz}$ vocalizations [19].

In contrast, high frequency ultrasonic vocalizations $(\sim 50 \mathrm{kHz})$ have been reported to be evoked during "positive emotional states", and induce and/or coincide with approach responses. The vocal presentations have been considered to be a direct behavioral expression of the relative hedonic valence of the environment at the time of the recordings (i.e., pleasant versus aversive) based on the generally held theory that animals approach positive hedonic stimuli and avoid or escape negative or aversive stimuli [20,21].

It should be remembered, as shown in figures 1 and 2, that a conclusion of a rodent subjective state similar to human reports of anxiety or fear associated with the high frequency vocalizations during $\mathrm{CO}_{2}$ is not consistent with the learned behaviors and stimulus-response contingencies associated with the ultrasonic vocalization, as corroborated by data appearing in peer-reviewed scientific journals. Vocalizations are spontaneous, invariant, apparently genetically, "hard-wired", and strain specific and do not rely on intervening factors involved in the behavioral assays described, below. These vocalizations represent the core survival reflex of the species.

The low frequency $(22 \mathrm{kHz})$ vocalizations are the frequencies associated with aggression, inescapable aversive stimuli, social defeat, and accordingly what would most likely constitute an anthropomorphic subjective state of "anxiety" [6,22,23]. Paradoxically, and in contrast with these "hypothesized" subjective states in rats, is the reported $22 \mathrm{kHz}$ vocalization in male rats during ejaculation [24]. However, this specific mating-behavior stress vocalization seems to be most suitably explained as appropriate to warn off all other male rat suitors in the area that might compete for mating rights and is intended to invoke the same fear response as if a real predator was in the area $[25,26]$. 
What must be kept in mind, however, is that it is vocalization within the high frequency bands of approximately $50 \mathrm{kHz}$ that were recorded in female rats during $\mathrm{CO}_{2}$ euthanasia. Mid-to-high frequency ultrasonic vocalizations of $40 \mathrm{kHz}$ have been recorded during separation of young rat pups from the dam [24]. The relative comparison between the $40 \mathrm{kHz}$ and the $22 \mathrm{kHz}$ vocalizations has been the subject of research for over three decades. Recent evidence reported by Blumberg \& Alberts [24], have provided demonstrative evidence that the $40 \mathrm{kHz}$ vocalization in pups and the $22 \mathrm{kHz}$ vocalization in adult rats are the acoustic by-product of a respiratory mechanism that enhances gas-exchange in the lungs during times of increased oxygen consumption. Blumberg \& Alberts [24] conclude that the two vocalizations (40 and $22 \mathrm{kHz}$ ) are produced by identical mechanisms and reflect identical physiological states, and are actually the same vocalization, albeit a different frequency based on maturation, alone. The high frequency ultrasonic vocalization recorded by Chisholm et al., [17] during euthanasia paradoxically include the 50 to $60 \mathrm{kHz}$ wavelength bands associated with anticipatory or actual reward stimuli, social interactions, and approach behaviors.

In a series of studies conducted by Dr. Jaak Panksepp and colleagues demonstrate that $50 \mathrm{kHz}$ ultrasonic vocalizations reflect a positive emotional state in rats $[27,28]$. Playbacks of these vocalizations are rewarding [17], and are considered to be positive events. Panksepp has suggested that the $50 \mathrm{kHz}$ vocalizations are evoked during "tickling" by the technician, during rough-and-tumble play with cohorts, and is akin to "laughter" in the rat [29]. These sounds are also evoked following administration of hedonically positive drugs of abuse, such as amphetamine [29], cocaine [30] and methylphenidate [31] as well as the direct dopamine agonist apomorphine [32]. Maier, Ma, Ahrens, Schallert and Duvauchelle [33] have also reported $50 \mathrm{kHz}$ vocalizations in rats evoked while self-administering cocaine in standard operant self-administration paradigms. Interestingly, rats also emit the $50 \mathrm{kHz}$ calls when exposed to a clean cage; male rats vocalize (including in this frequency range) more in bedded cages than in bare ones [34].

Brudzynski [35] has proposed that the $22 \mathrm{kHz}$ vocalization is induced by increases in cholinergic tone in the diencephalon and forebrain of the rat, and the $50 \mathrm{kHz}$ vocalizations are induced by increases in dopamine concentrations in the reward centers of the nucleus accumbens and basal ganglia. Adopting this more biologically based explanation, it may be most harmonious with the given vocalization data to conclude that the high ultrasonic vocalizations recorded by Chisholm et al., [17] during $\mathrm{CO}_{2}$ euthanasia was induced by increases in subcortical dopamine induced by handling by the technicians, placement into the clean vapor chamber, and the induction of dopamine release induced by the $\mathrm{CO}_{2}$ itself.

It has been established in humans that:

1. "Huffing", the intentional recirculation of air from paper or plastic bags filled with airplane glue, metallic paints [36,37] etc., and

2. Adolescents who use "crackers" to self-administer $\mathrm{CO}_{2}$ "whippet" containers (for dispensing whipped cream) to get a "high" [38] and

3. The self- inhalation of Nitrous oxide (NO) party balloons at "raves" [39] provide atmospheric conditions closely approximating those occurring in the rat in the euthanasia chambers. All adolescent abuse of such inhalants has been linked to the subjective state of being "high", or "euphoric". Clearly, based on the continued abuse of the inhalants by adolescents there is no obvious support that these anoxic events are aversive or anxiety provoking in human drug abusers. If Panksepp and colleagues' interpretation of the vocalization data are correct, then the ultrasonic vocalizations recorded by Chisholm et al., [17], demonstrate that $\mathrm{CO}_{2}$ exposure during euthanasia elicits an internal state more consistent with the evocation of rat "laughter", not anxiety.

In summary, the Chisholm et al., [17] report of high ultrasonic vocalizations emitted by female rats during $\mathrm{CO}_{2}$ euthanasia are more consistent with those conditions associated with approach behaviors in response to appetitive/pleasant stimuli. The anoxic conditions corresponding with verbal reports of "euphoria" or "high" in humans who abuse inhalants would seem to argue against a preemptory conclusion that the same conditions (anoxia) must necessarily represent an aversive situation in the rat. The $22 \mathrm{kHz}$ vocalizations generally correlated with anxiety, aggression, and escape in rats were not in the recorded wavelength band of 30 to $70 \mathrm{kHz}$ in the Chisholm et al., study [17]. Under the AVMA guidelines, "virtually all animals, being placed in a novel environment is stressful; therefore, a euthanasia approach that can be applied in familiar surroundings may help reduce stress (p. 14)".

Recently, some authors proposed a "two pronged" approach to $\mathrm{CO}_{2}$ euthanasia using a pre-exposure to vaporous inhalation anesthesia "to-effect" prior to exposure to $\mathrm{CO}_{2}$. Mice exposed to volatile anesthetic, isoflurane, produced ultrasonic calls with a peak frequency of $26.5 \mathrm{kHz}$. We propose, then, that including isoflurane exposure prior to the rodent $\mathrm{CO}_{2}$ protocol has the potential of inducing anxiety and distress that would not be present by $\mathrm{CO}_{2}$ exposures alone.

Accordingly, at present there exists no compelling evidence to suggest that the ultrasonic vocalizations evoked by rats undergoing $\mathrm{CO}_{2}$ euthanasia procedures is either directly aversive or has any aversive emotional-evoking response to living rodent cohorts 10 to 12 feet removed. Documented recordings of the ultrasonic vocalizations emitted by rodents during $\mathrm{CO}_{2}$ exposures are not consistent with "pain" or a "state of distress". We posit the supportive premise of our argument that retaining rodents in their home vivarium during end-of-study termination does not expose them to any alarm call or species-specific warning calls evoking anxiety or stress. The current approved policy is supported by critically relevant literature appearing in peer-reviewed scientific journals and is consistent with the ethical standards of the AVMA.

\section{Conditioned place aversion/preference}

The basic guiding principle of approach/avoidance behavior by the AVMA has been the foundation for standard place conditioning assays to assess the relative hedonic valence of interoceptive stimuli such as drugs and euthanasia gases, such as carbon dioxide.

While overt behavioral signs of distress have been reported in some studies, others have not consistently found these effects. Through preference and approach-avoidance testing, all inhaled agents currently used for euthanasia have been identified as being aversive to varying degrees. Aversion is a measure of preference, and while aversion does not necessarily imply that the experience is painful, forcing animals into aversive situations creates stress (AVMA Guidelines, left column, page 18)

Using preference and approach-avoidance testing, rats and mice show aversion to $\mathrm{CO}_{2}$ concentrations sufficient to induce 


\section{unconsciousness (AVMA Guidelines, left column, page 25)}

The experimental analysis of behavior associated with the assessment of the subjective hedonic valence continuum of euphoria/dysphoria do no support this premise of the AVMA guidelines. It is a categorical error to assume that aversive stimuli are not rewarding or that a preference for one stimulus condition implies euphoria. Drugs that can produce aversion responses can also induce preferences at the same doses [40]. Showing aversion to $\mathrm{CO}_{2}$ under a certain set of behavioral contingencies does NOT give supportive evidence that $\mathrm{CO}_{2}$ is dysphoric or induces distress.

As cited by the AVMA Policy Statement on Euthanasia, Leach, Bowell, Allan, \& Morton [41] showed conditioned place aversions induced by three different concentrations of carbon dioxide in rats and mice with 3-minute exposure periods. These authors concluded from these behavioral findings that the repeated exposures to carbon dioxide must have been based solely on escape behaviors of the mice and rats, defined as the reduced time spent in the gas-paired environments during gas-free test sessions and, therefore, $\mathrm{CO}_{2}$ is aversive in rodents. In the same year as the Leach et al., report was published, Funada, Sato, Makino and Wada [42] reported significant conditioned place preferences induced by exposures to 700 to $3200 \mathrm{ppm}$ toluene in mice in 20 minute sessions. The following year, Gerasimov, Collier, Ferrieri, Alexoff, Lee, Gifford and Balster [43] report similar place preferences induced by toluene in rats. Using similar airtight chambers, Lee, Gerasimov, Schiffer, and Gifford [44] clearly demonstrated strong place preferences induced by toluene vapors in both rats and mice. Importantly, these authors concluded that experiments with volatile stimuli (like toluene, $\mathrm{CO}_{2}$, etc.) were critically dependent on both the gas concentrations and the number of environment/vapor pairings. Leach et al., [41] used a single exposure period of 3 minutes for all gas concentrations tested and it is not clear what between-trial washout periods were used between gas and "normal air" pairings in the study.

The Leach et al., [41] data seem intuitively to support their conclusion, but when compared to the vast literature on conditioned place preferences induced by other interoceptive stimuli, like drugs, the disparate findings of what will and what will not induce place preferences reflect the general "paradox" of hedonic valence testing that was not addressed by Leach et al., $[41,45,46]$ or the AVMA, in its policy statement.

General learning theory focusing on conditioning of internal stimulus events, such as those induced by drug administrations, has identified that the subjective response to the drugs can be conditioned or be learned to produce aversions by the same drugs and specific drug doses that also produce approach behaviors (i.e., preferences; [40,47]). This hedonic paradox cannot be explained by secondary reinforcing effects, differential response topographies, differential basal homeostasis, or the rate of internal state changes [48]. Learning paradigms involve more than a simple Pavlovian stimulus-response neural engram.

Learning paired associations between repeated presentations of a stimulus (gas and environmental stimuli) involves more than a simple reflexive arc. It has been repeatedly demonstrated that animals can be conditioned to initiate and maintain the self-delivery of electric shock [49-51] but likely few would ever correspondingly assert that electric shocks are pleasurable. Using similar conditioned place preference assays as Leach et al., [41] it has been widely reported by many laboratories that ethyl alcohol, one of the world's most abused drugs, induces place aversions in rodents similar to $\mathrm{CO}_{2}$ in the Leach study [52-54]. Do we conclude that rats find alcohol intoxication aversive?

Intravenous pentylenetetrazole (Metrazole ${ }^{\mathrm{TM}}$, aka PTZ) administrations were used in psychiatry in the 1950s to induce convulsions in depressed patients similar to insulin-induced and Electroconvulsive Therapies (ECT) [55]. A series of daily injections were used to induce tonic/clonic convulsions with the intention of alleviating the patient from the depths of depression. The irreversibility and the lack of control of the duration of seizures by chemotherapeutic-inductions using insulin and PTZ soon moved the therapy to ECT. Rodin and colleagues reported the clinical observations that many of the PTZ-treated patients reported an intense state of anxiety induced by the drug $[48,56]$. In the following years animal models of anxiety were developed using PTZ as the positive control drug to induce the "anxiety like" state in rodents [6]. Similar to Leach et al., [41,45,46] the conditioned place preference assay has been used to assess the relative hedonic valence of PTZ (anxiety) in rats. A conditioned place preference was induced by the proconvulsant, PTZ, in rats [57] do we conclude from these findings that rats prefer pre-ictal or ictal events?

In its comparative evaluations between the subjective effects of $\mathrm{CO}_{2}$ in humans and rats, the AVMA cites, in part, previous work by Argyropoulos, Bailey, Hood, Kendrick, Rich, Laszlo, Nash, Lightman, and Nutt [58]. In this report 14 male human subjects were exposed to a brief exposure of $35 \% \mathrm{CO}_{2}$ in a laboratory setting. The male subjects were NOT excluded for "smoking history", as long as they did not smoke more than 5 cigarettes a day. The subjects were unaware of the sequence of the gas delivery (i.e., air or $\mathrm{CO}_{2}$, in order to reduce expectations). Subjects were asked to inhale a single breath test of a gas mixture (room air or $35 \% \mathrm{CO}_{2}$ ), and to hold the breath for $4 \mathrm{sec}-$ onds and then, to breath normally. This study is cited by opponents of the use of $\mathrm{CO}_{2}$ in rodents since it is believed to demonstrate that the single breath test confirmed a sense of distress in human subjects. In spite of the potential selection criteria errors of using cigarette smoking male subjects and limiting the analysis to a single breath-hold test, it seems to be ignored that the salivary cortisol response and heart rates were not correlated to the visual analog scale of "fear" measured in the study. While visual analog scale measures of "fear" peaked at the time of the $\mathrm{CO}_{2}$ breath hold, the cortisol response was not demonstrated for 20 minutes following the exhaled gas. In spite of changes in the visual analog scale representing "fear" response during the novel breath hold, the heart rate of the experimental subjects at the time of peak "fear" actually decreased when compared to air holding samples, and the systolic blood pressure transiently increased by 24 mmHg. Is "fear" or "anxiety" usually correlated with decreases in heart rates and non-correlated changes in cortisol in humans? Could these results be more indicative of a subjective state change in relation to unexpected and sudden novelty within the experimental paradigm used by these authors?

Recently, Hayen, Hergstad, Wiech and Pattinson [59] at the University of Oxford, and Ainsworth, Marshall, Meron, Baldwin, Chadwick, Munafo and Garner [60] at the University of Southampton in the United Kingdom examined differential $\mathrm{CO}_{2}$ inhalation parameters in human subjects. The Southhampton laboratory supplied 20 minutes of $7.5 \% \mathrm{CO}_{2}$ to control subjects and two groups of differentially trained "meditation" subjects (focused attention and open monitoring). Most interesting to the present discussion, these authors reported that "participants in each group reported lower levels of anxiety 
following $\mathrm{CO}_{2}$ challenge relative to their own baseline values and the magnitude of this reduction did not differ across groups in the study" (Section 3.2.).

In the Oxford study, Hayen, Hergstad, Wiech and Pattinson [59] were interested in subjective mood changes induced by $\mathrm{CO}_{2}$ in humans that may be combined with controlled gas delivery for Functional Magnetic Resonance Imaging (FMRI) and the Blood Oxygen Level Dependent Response (BOLD) uses as a source of contrast in the FMRI. The subjective effects of stabilized (isocapnia) and mild hypercapnia (increased end tidal $\mathrm{CO}_{2},\left[\mathrm{P}_{\mathrm{ET}} \mathrm{CO}_{2}\right]$ ) induced by fine-tuning on a breath-by-breath basis of adjusting $\mathrm{CO}_{2}$ in the inspired gas mixtures were measured in initially naïve human patients conditioned to the experimental presentations - these conditions may be more similar to the Leach et al., [41] studies since all experimental subjects had prior experience with $\mathrm{CO}_{2}$ before subjective scoring measures were used. In the Oxford study, the authors reported that the subjective ratings of "unpleasantness" of hypercapnia showed no main effect of $\mathrm{CO}_{2}(\mathrm{p}=0.178)$, and no significant interaction effects with the four trials of the exposures $(\mathrm{p}=0.602)$.

The main findings in both 2015 studies confirm the variability in the subjective response to $\mathrm{CO}_{2}$ exposures (in humans and rats) and appear to admonish the use of the Leach et al., $[41,45,46]$ as a valid argument to deter a legitimate and scientifically sound form of rodent euthanasia. While Leach et al., $[41,61]$ data are noteworthy, the data must be viewed with the knowledge that only a single exposure period ( 3 minutes) was used in the "behavioral learning paradigm". Using similar techniques Boulanger-Bertolus, Nemeth, Makowska, and Weary [62] have more recently shown that alternative anesthetics, isoflurane [63] and sevoflurane, also produce aversions in rats. Learning theorists, in general, have shown that such repeat-exposure conditioning models are highly influenced by the current and past environmental/behavioral contingencies in effect at the time of exposures. Such data do not supply definitive support for claiming that $\mathrm{CO}_{2}$ exposures induce a subjective state in rats similar to those that induce a verbal report of "anxiety" in humans.

Interestingly, the National Institute on Drug Abuse has concluded that inhalant abusers (nitrous oxide, $\mathrm{CO}_{2}$, nitrites [amyl nitrite] and even butane) demonstrate slurred speech, lack of coordination, and dizziness, but also report feeling of euphoria, not anxiety. Repetitive, controlled access to oxygen deprivation states induced by human inhalation of $\mathrm{CO}_{2}$, nitrous oxide, is demonstrative evidence that these dyspneic states are not inherently "distressful" or "anxiety-inducing". Humans seek out oxygen deprivation states they are self-driven to continue these forms of oxygen deprivation. Therefore, there does not seem to be any credible data to conclude that such atmospheric conditions should provide any substantially discordant (e.g., aversive) subjective state in rats under the "euthanasia" scenario with $\mathrm{CO}_{2}$.

Considerable caution should be exercised by researchers utilizing an "aversion" procedure in an attempt to demonstrate the specificity of $\mathrm{CO}_{2}$ exposures on behavior. Rodents show both aversion and preference to the same experimental condition. Humans seek out oxygen deprivation states there is no logical extension of these findings to presume that rats are in a state of anxiety, pain, or distress when exposed to the same dyspneic environment.

\section{Pheromones/airborne chemical messages}

In accordance with the definition first proposed by von Frisch [64], we call substances which communicate the presence of danger, "alarm substances" (Schreckstoff), provided that they are produced by members of the same species [65]. We define the communication of danger by observing the response of animals to the substance under investigation. According to Pfeiffer [16] odor-dependent escape reactions have been described in several animal species, and may be caused by either the repellant odor of an enemy, or by a specific alarm substance given off by an individual of the same species when attacked.

While impressions may be widespread that these chemotropic airborne messages (e.g., aggregation, attractor pheromones) waft across acres or miles of open fields setting into motion a number of fixed action patterns in a given species' cohorts, it is generally accepted that the majority of pheromones identified have limited and minimal effective receptive fields (e.g., primer, territorial, landmarking, alarm). The mere presence of an air-borne chemical in the immediate presence of an animal is not demonstrative evidence of its efficacy to induce behavioral change or to capture stimulus control of ongoing behavior of another animal in close proximity [65].

Pfeifer [66] described a series of studies on the presence of alarm substances in ants and found that ants had to pass over dead bodies or have direct contact with the surface at which dead ant bodies were removed in order for the ants to demonstrate behavioral changes suggesting the presence of an alarm substance. Von Frisch [64] reported the same finding in bees, in which alarm substances were transmitted only during the "waggle dance" of hive cohorts or by an individual bee passing over a dead bee from its own colony.

Ernest Abel, in a series of studies [67-69], examined the structure and function of rat alarm substances using the porsolt inescapable water swim test [70,71], and has clearly identified the limits of such chemotropic substances in laboratory rats. In the course of this research, Abel has reported that rat alarm substances are low volatile molecules. In the Abel swim studies, rats had to be placed into the water before alarm substances could function to activate behavioral changes. Suspending rats over a paper towel soaked in the water from the forced swim column (of less than 1 inch) fails to induce behavioral change. Placing a rat on a metal screen suspended just over the water surface also failed to induce behavioral changes in the rats [67]; rats had to physically be in the water in order to demonstrate behaviors indicative of perception of the low volatile alarm substances deposited by previous rats in distress (but see [72] ).

Based on the seminal work of Dr. Abel and colleagues, it may be tempting to make assumptions or over generalize the presence or activity of alarm substances by all rats under all stressful conditions. Tachibana et al., [73] using an identical forced swim apparatus and stress methodology as Dr. Abel, failed to demonstrate the presence of any behavioral change in Sprague-Dawley rats purchased from two Japanese suppliers (Charles River, Japan and SLC, Inc. Japan). Even when exposures were quadrupled to those described by Abel in previous work, Tachibana, Yoko, and Yoshino failed to find any evidence of an alarm substance in the Japanese Sprague-Dawley rat lines.

In two other experiments, the subjective or interoceptive stimulus properties of alarm substances were tested in another "animal model of anxiety" - the drug discrimination, operant, two-lever choice paradigm using the proconvulsant, Pentylenetetrazole (PTZ). As described above, PTZ has been used as an animal analogue of human anxiety $[61,74]$. In the first study, Gauvin \& Holloway [54], trained rats to discriminate the presence versus absence of $15 \mathrm{mg} / \mathrm{kg} \mathrm{PTZ}$, in test sessions rats were exposed to the smell, sight, and sounds of a feral 
cat within the restricted confines of a shielded exposure chamber. A review of the existing literature base on cat-rat interactions clearly identified the fact that species-specific-defense-reactions that are associated with "alarm substance" deposits by a rat required more than just the mere sight and smell of a cat. In naturalistic settings it is the "pouncing action" of the cat and the presence of rat cohorts that induces the behavior and "scent release". In a series of studies it was determined that the laboratory conditions needed to induce responding on the PTZ-appropriate lever during cross-generalization tests within this animal model of human "anxiety", was twofold: 1) the cat required pretreatment with "catnip", to induce a state of excitement in the cat, and 2) a cohort of rats $(n=4)$ needed to be placed in the exposure chamber together during cat-rat exposures. It is the presence of the "pouncing cat" and the "panicked cohorts" that facilitate release of the alarm substance under these conditions - a scenario of "mass or aggregrate contagion".

Behavioral data from these studies indicated that rats emitted $>90 \%$ of responding on the "anxiety-associated" lever when removed and tested following mass/aggregate exposure of four rats to a catnip-treated, pouncing cat. The drug discrimination model utilized in these experiments was accordingly demonstrated to be sensitive to the "anthropomorphic" concept of a subjectively-similar "anxiety" situation in rats.

To further test the applicability of the drug discrimination paradigm to assess interoceptive states induced by alarm substances to situations characterized by humans as "anxiety", Gauvin et al., [75] trained another group of Sprague-Dawley rats to discriminate the presence and absence of anxiety using three drug conditions: chlordiazepoxide (Librium ${ }^{\mathrm{TM}}$; relaxed state), saline (neutral state), and PTZ (anxiety state). In this study, alarm substance release was induced by placing a cohort of rats into the test chamber and exposing them to foot shock just prior to placing a different cohort of drug discrimination trained rats into the chamber under "saline treated" conditions. It was hypothesized that if alarm substance was deposited by electrical floor grid stimulation, then saline treated rats in the presence of alarm substance residues would induce a subjective state of "anxiety" and the animals would then elicit responding on the "anxiety-appropriate" lever (PTZ lever). Under this condition, rats engendered only $50 \%$ of their total session responding on the saline and PTZ levers no responding on the relaxed-appropriate, CDP lever occurred in these tests. Investigators then took another set of similarly trained rat cohorts, placed them under inescapable floor shock presentations and then removed blood and deposited the blood under the flooring of the operant chambers in the hopes of providing sufficient olfactory cues to engender PTZ-appropriate responding. The blood under the grated floor was not sufficient to increase the percentage of total session responding on the PTZ-appropriate lever. However, when the blood aliquot was placed inside the test environment in a position that allowed direct nose to blood contact, all rats engendered $100 \%$ of lever press responding on the anxiety-appropriate lever.

These studies corroborated Dr. Abel's conclusion that alarm substances deposited by rats are extremely low volatile substances, that do not migrate (aerosolize) more than one or two inches from the deposited substance, and that rats require extremely close contact in order to perceive the alarm stimulus. Similar to Dr. Abel's findings, Gauvin et al., [74] tests of blood and alarm substance from Wistar rats failed to induce behavioral changes suggestive of "anxiety-like" states in Sprague-Dawley rats.
In conclusion, the literature on alarm substances in rats clearly demonstrates that some rat species emit an alarm substance when stressed, as is "anthropomorphically" proposed to occur in the standard $\mathrm{CO}_{2}$ euthanasia scenario. However, that alarm substance is not either generated or deposited by all rats equivalently, and is of such low volatility that even one inch of air space between the rat's nose and the deposited alarm substance diminishes its ability to modify ongoing behaviors. Dr. Abel's research findings indicate that paper towels soaked in alarm substance deposited in water and placed within 1 inch of rat cohorts were insufficient to induce anxiety-like behavioral changes. This was supported by Gauvin \& Holloway [54] and Gauvin and colleagues [52] research showing that blood-borne alarm substances positioned less than 1 inch below the rats would not induce "anxiety-like" responding in another behavioral assay sensitive to this construct in the rat.

The understanding of the conceptual framework surrounding pheromones must be positioned based on a thorough reading of the limitations and scope of such air-borne chemical messages before adopting generalized rules to govern intentionally and carefully-developed standard operating procedures in independent test facilities. Within a standard rodent room the environmental conditions of negative air flow, and high volume circulation ( $>10$ exchanges per hour), the expansive space between housed cohorts and euthanasia equipment, and the olfactory scent masking provided by the human technicians, the prior use of cleaning agents, etc., do not provide an atmosphere conducive to support the hypothesis that relatively weak and localized alarm substances can be detected by home cage cohorts at the time of euthanasia.

In view of the existing literature appearing in peer-reviewed scientific journals, rational and compelling arguments advocating the potential influence of pheromones as mediators of $\mathrm{CO}_{2}$-induced anxiety states in rats appears to be anthropomorphically driven. The low volatility of these airborne chemical messages does not provide convincing evidence that rat cohorts in the vivarium at the time of $\mathrm{CO}_{2}$ euthanasia are exposed to the pheromones or are functionally affected by the release of the pheromones within the euthanasia chamber just feet away from their home cage.

\section{Conclusion}

The National Research Council's Committee on pain and distress in laboratory animals [76] concluded that there is no evidence that all normal activities of a species are based on specific behavioral needs or motives whose blocking or presentation causes distress (p. 49). The mere presence of vocalizations, chemical residues, or simple proximity to an event does not necessarily demonstrate or suggest the presence of a subjective perception of an emotional state that is described by humans under similar conditions as anxiety or fear. While it is imperative for all scientists involved in research to stay abreast of the current and historically-relevant literature appearing in peer reviewed scientific journals, it is also important to alleviate any fears or concerns of the technical staff that handles the animals or is present in the laboratory during the conduct of euthanasia procedures.

Under the AVMA guidelines " if welfare is defined solely in terms of an animal's subjective experience, euthanasia may be warranted even if the animal is not showing signs of suffering at the present time and if there is some commitment to avoid harm (p. 8)". The AVMA standard of terminating healthy animals under such circumstances, while unpleasant and morally challenging, is a practical necessity. 
The AVMA recognizes that in some cases in laboratory situations, for example, "where animals are employed as research subjects and death may be a terminal point, animal welfare considerations are balanced against the merits of the experimental design and merits of the research (p. 9)". In such cases, ensuring the respectful and humane treatment of research animals will be largely determined by the anatomy, biology, and ethology of the animal species as well as the review of such procedures by the Institutional Animal Care and Use Committees (IACUC). Under the conditions set forth by the AVMA, the technicians in research laboratories around the world should be confident that the physiology and biological make-up of rats are sufficiently different from humans. There is no credible evidence that the psychological response to the laboratory-bred rats' perception of environmental conditions involved in end-of-study euthanasia is any different than their normal day-to-day living experiences within the same laboratory.

\section{References}

1. Szil A, Rohlich P (1992) Two cone types of rat retina detected by anti-visual pigment antibodies. Exp Eye Res 55: 47-52.

2. Mohler EG, Ding Z, Rueter LE, Chapin D, Young D, et al. (2015) Crosssite strain comparison of pharmacological deficits in the touchscreen visual discrimination test. Psychopharmacology (Berl) 232: 4033-4041.

3. Redfern WS, Storey S, Tse K, Hussain Q, Maung KP, et al. (2011) Evaluation of a convenient method of assessing rodent visual function in safety pharmacology studies: effects of sodium iodate on visual acuity and retinal morphology in albino and pigmented rats and mice. J Pharmacol Toxicol Methods 63: 102-114.

4. Thomas BB, Samant DM, Seiler MJ, Aramant RB, Sheikholeslami S, et al. (2007) Behavioral Evaluation of Visual Function of Rats Using a Visual Discrimination Apparatus. J Neurosci Methods 162: 84-90.

5. Wong AA, Brown RE (2006) Visual detection pattern discrimination and visual acuity in 14 strains of mice. Genes Brain Behav 5: 389-403.

6. Kumar G, Talpos J, Steckler T (2015) Strain-dependent effects on acquisition and reversal of visual and spatial tasks in a rat touchscreen battery of cognition. Physiol Behav 144: 26-36.

7. Prusky GT, Douglas RM (2005) Vision. In: Whishaw IQ, Kolb B (eds.) The behavior of the laboratory rat. Oxford University Press, New York, USA.

8. Abel PL, Olavarria JF (1994) The collosal pattern in striate cortex is more patchy in monocularly enucleated albino than pigmented rats. Neurosci Lett 204: 169-172.

9. Jeffery G (1998) The retinal pigment epithelium as a developmental regulator of the neural retina. Eye 12: 499-503.

10. Lund RD, Lund JS, Wise RP (1974) The organization of the retinal projection to the dorsal lateral geniculate nucleus in pigmented and albino rats. J Comp Neurol 158: 383-403.

11. Abadi R, Dickinson CM, Pascal E, Papas E (1990) Retinal image quality in albinos. A review. Ophthalmic Paediatr Genet 11:171-176.

12. Birch D, Jacobs GH (1977) Effects of constant illumination on vision in the albino rat. Physiol Behav 19: 255-259.

13. Nitschke W (1982) Acoustic Behavior in the Rat: Research, Theory, and Application. Praeger Publishers, Santa Barbara, California, USA.

14. Borszcz GS (2006) Contribution of the Ventromedial Hypothalamus to Generation of the Affective Dimensions of Pain. Pain 123: 155-168.

15. Sanders J, Weisz DJ, Yang BY, Funk K, Amirali A (2001) The mechanism of ultrasonic vocalization in the rat. Neuroscience Abstracts 27: 88.19 .
16. Nyby J, Whitney G (1998) Ultrasonic communication of adult myomorph rodents. Neurosci Biobehav Rev 2: 1-14.

17. Chisholm J, De Rantere D, Fernandez NJ, Krajacic A, Pang DS (2013) Carbon dioxide, but not isoflurane, elicits ultrasonic vocalizations in female rats. Lab Animal 47: 324-327.

18. Sadananda M, Wohr M, Schwarting RK (2008) Playback of $22 \mathrm{kHz}$ and $50 \mathrm{kHz}$ ultrasonic vocalizations induces differential c-fos expression in rat brain. Neurosci Lett 435: 17-23.

19. Berger AL, Williams AM, McGinnis MM, Walker BM (2013) Affective Cue-Induced Escalation of Alcohol Self-Administration and Increased 22-kHz Ultrasonic Vocalizations during Alcohol Withdrawal: Role of Kappa-Opioid Receptors. Neuropsychopharmacology 38: 647-654.

20. Bindra D (1974) A motivational view of learning, performance, and behavior modification. Psychol Rev 81: 199-213.

21. Hearst E, Jenkins HM (1974) Sign-tracking : the stimulus-reinforcer relation and directed action. Psychonomic Society, Texas, USA.

22. Litvin Y, Blanchard DC, Blanchard RJ (2007) Rat 22kHz ultrasonic vocalizations as alarm cries. Behav Brain Res 182: 166-172.

23. Portfors CV (2007) Types and functions of ultrasonic vocalizations in laboratory rats and mice. J Am Assoc Lab Anim Sci 46: 28-34.

24. Blumberg MS, Alberts JR (1991) On the significance of similarities between ultrasonic vocalizations of infant and adult rats. Neurosci Biobehav Rev 15: 383-390.

25. Anisko JJ, Suer SF, McClintock MK, Adler NT. 1978. Relation between $22-\mathrm{kHz}$ ultrasonic signals and sociosexual behavior in rats $\mathrm{J}$ Comp Physiol Psychol 92: 821-829.

26. Barfield RJ, Geyer LA (1975) The ultrasonic postejaculatory vocalization and the postejaculatory refractory period of the male rat. Journal of Comparative and Physiological Psychology 88: 723-734.

27. Burgdorf J, Panksepp J (2001) Tickling induces reward in adolescent rats. Physiol Behav 72: 167-173.

28. Burgdorf J, Panksepp J, Moskal JR (2011) Frequency-modulated 50kHz ultrasonic vocalizations: a tool for uncovering the molecular substrates of positive affect. Neurosci Biobehav Rev 25: 1831-1836.

29. Iacobucci P, Colonnello V, Fuchs T, D’Antuono L, Pankseep J (2013) Differential ultrasonic indices of separation distress in the presence and absence of maternal cues in infant rats bred for high and low positive social affect. Acta Neuropsychatr 25: 289-296.

30. Barker DJ, Root DH, Ma S, Jha S, Megehee L, et al. (2010) Dose-dependent differences in short ultrasonic vocalization emitted by rats during cocaine self-administration. Psychopharmacology (Berl) 211: 435-442.

31. Simola N, Fenu S, Costa G, Pinna A, Plumitallo A, et al. (2012) Pharmacological characterization of $50-\mathrm{kHz}$ ultrasonic vocalizations in rats: comparison of the effects of different psychoactive drugs and relevance in drug-induced reward. Neuropharmacology 63: 224-234.

32. Williams SN, Undieh AS (2010) Brain-derived neurotrophic factor signaling modulates cocaine induction of reward-associated ultrasonic vocalization in rats. J Pharmacol Exp Ther 332: 463-468.

33. Maier EY, Ma ST, Ahrens A, Schallert TJ, Duvauchelle CL (2010) Assessment of Ultrasonic Vocalizations During Drug Self-Administration in Rats. J Vis Exp 41.

34. Natusch C, Schwarting RK (2010) Using bedding in a test environment critically affects $50-\mathrm{kHz}$ ultrasonic vocalization in laboratory rats. Pharmacol Biochem Behav 96: 251-259.

35. Brudzynski SM (2009) Communication of adults rats by ultrasonic vocalization: biological, sociobiological, and neuroscience approaches. ILAR J 50: 43-50. 
36. Baird CA, Furek MW (2012) Adolescents and inhalant abuse: how huffing affects the myelin sheath. J Addict Nurs 23: 129-131.

37. Baydala L (2010) Inhalant Abuse. Paediatr Child Health 15: 443-448.

38. Winston A, Kanzy A, Bachuwa G (2015) Air duster abuse causing rapid airway compromise. BMJ Case Rep.

39. Bäckström B, Johansson B, Eriksson A (2015) Death by nitrous oxide. J Forensic Sci. 60: 1662-1665.

40. Van der Kooy D, Swerdlow NR, Koob GF (1983) Paradoxical reinforcing properties of apomorphine: Effects of nucleus accumbens and area postrema lesions. Brain Research 259: 111-118.

41. Leach MC, Bowell VA, Allan TF, Morton DB (2002) Aversion to gaseous euthanasia agents in rats and mice. Comp Med 52: 249-257.

42. Funada M, Akitake Y, Aoo N (2008) Evaluation of the rewarding effects of drug by conditioned place preference (CPP) paradigm: properties of volatile organic solvents and uncontrolled newly-abuse drugs. Nihon Arukoru Yakubutsu Igakkai Zasshi 43: 691-696.

43. Gerasimov MR, Collier L, Ferrieri A, Alexoff D, Lee D, et al. (2003) Toluene inhalation produces a conditioned place preference in rats. Eur J Pharmacol 477: 45-52.

44. Lee DE, Gerasimov MR, Schiffer WK, Gifford AN (2006) Concentration-dependent conditioned place preference to inhaled toluene vapors in rats. Drug Alcohol Depend 85: 87-90.

45. Leach MC, Bowell VA, Allan TF, Morton DB (2002) Degrees of aversion shown by rats and mice to different concentrations of inhalational anaesthetics. Vet Rec 150: 808-815.

46. Leach MC, Bowell VA, Allan TF, Morton DB (2004) Measurement of aversion to determine humane methods of anaesthesia and euthanasia. Anim Welf 13: 77-86.

47. Goudie AJ (1987) Aversive stimulus properties of drugs: The conditioned taste aversion paradigm. In: Greenshaw AJ, Dourish CT (eds.) Experimental Psychopharmacology. Humana Press, New York, USA. Pg no: 341-391.

48. Rodin E, Rodin M, Lavine L (1979) Electroclinical and ultrastructural changes associated with subconvulsant doses of pentylenetetrazole. Exp Neurol 64: 386-400.

49. McKearney JW (1968) Maintenance of responding under a fixed-interval schedule of electric shock presentation. Science 160: 1249-1251.

50. McKearney JW (1969) Fixed-interval schedules of electric shock presentation: extinction and recovery of performance under different shock intensities and fixed-interval durations. J Exp Anal Behav 12: 301-313.

51. McKearney JW (1970) Responding under a fixed-ratio and multiple fixed-interval fixed-ratio schedules of electric shock presentations. J Exp Anal Behav 14: 1-6.

52. Gauvin DV, Baird TJ, Briscoe RJ (2000) Differential development of behavioral tolerance and the subsequent hedonic effects of alcohol in AA and ANA rats. Psychopharmacology (Berl) 151: 335-343.

53. Gauvin DV, Briscoe RJ, Goulden KL, Holloway FA (1994) Aversive attributes of ethanol can be attenuated by dyadic social interaction in the rat. Alcohol 11: 247-251.

54. Gauvin DV, Holloway FA (1991) Cross-generalization between an ecologically relevant stimulus and a pentylenetetrazole-discriminative cue. Pharmacology Biochemistry and Behavior 39: 521-523.

55. CHOPRA BL (1946) A case of acute melancholia treated with cardiazol and insulin. Ind Med Gaz 81: 82.

56. Rodin E, Onuma T, Wasson S, Porzak J, Rodin M (1971) Neurophysiological mechanisms involved in grand mal seizures induced by Metrazo and Megimide. Electroencephalogr Clin Neurophysiol 30: 62-72.
57. Gauvin DV, Dormer KN, Holloway FA (1991) Pentylenetetrazole can induce a conditioned place preference. Pharmacol Biochem Behav 40: 987-990.

58. Argyropoulos SV, Bailey JE, Hood, SD, Kendrick AH, et al. (2002) Inhalation of $35 \% \mathrm{CO}(2)$ results in activation of the HPA axis in healthy volunteers. Psychoneuroendocrinology 27: 715-729.

59. Hayen A, Herigstad M, Wiech K, Pattinson KTS. 2015. Subjective evaluation of experimental dyspnoea--effects of isocapnia and repeated exposure. Respir Physiol Neurobiol 208: 21-28.

60. Ainsworth B, Marshall JE, Meron D, Baldwin DS, Chadwick P, et al. (2015) Evaluating psychological interventions in a novel experimental human model of anxiety. J Psychiatr Res 63: 117-122.

61. Lal H, Emmett-Oglesby MW (1983) Behavioral analogues of anxiety. Animal models. Neuropharmacology 22: 1423-1441.

62. Bertolus JB, Nemeth G, Makowska IJ, Weary DM (2015) Rat aversion to sevoflurane and isoflurane. Applied Animal Behaviour Science 164: $73-80$.

63. Wong D, Makowska IJ, Weary DM (2012) Rat aversion to isoflurane versus carbon dioxide. Biol Lett 9: 1-4.

64. Von Frisch K (1941) Über einen Schreckstoff der Fischhaut und seine biologische Bedeutung. Zeitschrift für vergleichende Physiologie 29: 46-145.

65. Wyatt TD (2014) Pheromones and animal behavior: Chemical signals and signatures. Cambridge. Cambridge University Press, London, UK.

66. Pfeiffer W (1963) Alarm Substance. Experientia19: 113-123.

67. Abel EL (1991) Alarm substance emitted by rats in the forced-swim test is a low volatile pheromone. Physiol Behav 50: 723-727.

68. Abel EL (1991) Gradient of alarm substance in the forced swimming test. Physiol Behav 49: 321-323.

69. Abel EL (1993) Strain differences in convulsions induced by alarm substance. Pharmacol Biochem Behav 45: 561-563.

70. Porsolt RD, Bertin A, Jalfre, M (1977) Behavioral despair in mice: a primary screening test for antidepressants. Arch Int Pharmacodyn Ther 229: 327-336.

71. Porsolt RD, Anton G, Blavet N, Jalfre M (1978) Behavioral despair in rats: a new model sensitive to antidepressant treatments. Eur J Pharmacol 47: 379-391.

72. Hauser R, Marczak M, Karaszewski B, Wiergowski M, Kaliszan M, et al. (2008) A preliminary study for identifying olfactory markers of fear in the rat. Lab Animal 37: 76-80.

73. Tachibana T, Yoko A, Yoshino H (1996) Cases of Sprague-Dawley Rats Which Did Not Respond to an 'Alarm Substance' in the Forced Swimming Test. Psychol Rep 79: 1007-1018.

74. Gauvin DV, Briscoe RJ, Baird TJ, Vallett M, Holloway FA (1997) The paradoxical hedonic valence of acute ethanol withdrawal (hangover) states in rats: Place and taste conditioning. Alcohol. 14: 261-268.

75. Gauvin DV, Briscoe RJ, Baird TJ, Vallett M, Carl KL, et al. (1996) Three-choice chlordiazepoxide, saline, pentylenetetrazole discrimination in rats: Cross generalization between drug and (Oflactory) alarm substance stimuli. Exp Clin Psychopharmacol 4: 373-378.

76. National Research Council (US) Committee on Recognition and Alleviation of Pain in Laboratory Animals (2009) Recognition and Alleviation of Pain in Laboratory Animals. National Academies Press, Washington DC, USA. Pg no: 341-391. 